\title{
Arteriographic view of treatment to achieve regression of coronary atherosclerosis and to prevent plaque disruption and clinical cardiovascular events
}

\author{
B Greg Brown, Xue-Qiao Zhao, Dianne E Sacco, John J Albers
}

Treatments designed to diminish the clinical impact of coronary artery disease (CAD) have two fundamental goals. The first goal is to reduce the symptomatic limitations imposed by an obstructed arterial blood supply which is unable to meet the peak oxygen demands of the myocardium. Vascular supply may be insufficient because of a fixed flow-limiting coronary narrowing, abnormal epicardial vessel tone, intermittent arterial vasospasm, microvascular dysfunction, or inadequate development of collateral vessels. Certain medical approaches presently palliate symptoms by favourably altering the imbalance between oxygen supply and demand. The goal of symptom relief may, alternatively, be attained through more direct structural or physiological changes or both that improve the diminished vascular flow reserve. These include relaxation of excess vasoconstrictor tone, development of collateral vessels, or reduction ("regression") in the severity of flow-limiting stenosis. Regression has not been universally accepted as a possible mechanism for symptom relief. We review the evidence supporting the occurrence of regression and its promotion by lipid lowering.

The second fundamental goal of treatment in CAD is to prevent the anticipated progression to a clinical event such as cardiac death, non-fatal myocardial infarction, or worsening angina requiring bypass surgery or angioplasty. Such ischaemic events commonly occur suddenly (even after a recent "perfectly normal" treadmill test) suggesting an abrupt transition in the severity of arterial obstruction. We also discuss the ways in which structural disruption of the atherosclerotic plaque may lead to abrupt worsening of arterial obstruction and, commonly, a clinical event. New data are presented that indicate a linkage between lipid lowering and stabilisation of plaque structure. They support the hypothesis that lipid-lowering treatment prevents clinical events by causing selective regression of the lipid-rich subgroup of plaques which are vulnerable to fissuring, ulceration, and haemorrhage.

Medicine, Cardiology

Division, University of Washington School of Medicine, Seattle, Washington, USA

B G Brown

$\mathrm{X}-\mathrm{Q}$ Zhao

D E Sacco

$\mathrm{J} J$ Albers

Correspondence to

Dr B Greg Brown

Cardiology RG-22,

University of Washington,

Seattle, WA 98195, USA.
Arteriographers occasionally observe the reopening of a previously obstructed coronary artery, presumably by the lysis of an occlusive thrombus. Furthermore, ordinary lesions are sometimes seen to improve in a later arteriogram. Thus the important question is not "does it happen clinically?" (it does), but "can we promote regression of sufficiently grea magnitude and frequency so that it favourably alters the clinical course of the disease?" As yet there is not a consensus but encouraging evidence is emerging.

\section{EXPERIMENTAL OBSERVATIONS}

Convincing evidence that atherosclerosis care regress with lipid lowering has come fromi studies in cholesterol-fed primates. ${ }^{1-5}$ Afte 5 ? prolonged exposure to a cholesterol-ric "atherogenic" diet there are large increases in coronary artery collagen $(3 \times)$, elastin $(4 \times)+\infty$ and cholesterol $(7 \times$, mostly esterified)옹 Studies of plaque lipid ${ }^{4}$ suggest that choles $=$ terol ester, transported to the intima bi plasma lipoproteins, accumulates first in foan cells and as interstitial droplets. After 24-30 months foam cells may undergo necrosis and release their lipid content. Macrophage-foano cells, derived from circulating monocytes, can also release proteolytic enzymeso Gradually, cholesterol accumulates deep in the intima in the core lipid region of the plaque as ester droplets and monohydrateक crystals intermixed with necrotic cellula? debris and coagulation products. When the animals are returned to a vegetarian "regres sion" diet, serum cholesterol falls quickly to normal $(3.6 \mathrm{mmol} / \mathrm{l})$ and the arterial lipid and connective tissue changes partially regres 8 over 20-40 months. Collagen does no decrease much from its peak value $(-20 \%)$ but elastin $(-50 \%)$ and cholesterol $(-60 \%$ ) mostly esterified) do. Histological measure $\frac{O}{3}$ ments show that plaque size is reduced. ${ }^{24}$

EVIDENCE OF REGRESSION IN PATIENTS

Methods to detect regression of atherosclero sis in patients with coronary heart disease have, necessarily, been indirect. Improvements in the severity of angina may indicate plaque regression; it may, however, occur by various other mechanisms including the developmen $\$$ of collateral vessels, myocardial necrosis, o exercise conditioning. Myocardial perfusion ${ }^{+}$ imaging ${ }^{7}$ has some of the same limitations and shows little or nothing about change in mild obstructive lesions. The coronary arteriogran has been and remains the principal methoo for assessing change in disease. It provides aq accurate estimate of the severity of lumina narrowing; however, it does not tell a greas deal about the complex interplay of structurat and physiological processes in the arterial wall that contribute to the degree of obstruction. There is, in some circles, a growing perception that certain morphological features of the 
plaque permit inferences about wall properties, ${ }^{8}$ and that the lumen diameter response to certain vasoactive drugs provides information on the functional health of the artery. Intravascular ultrasound ${ }^{8}$ may be diagnostically useful, and will undoubtedly improve our understanding of the relation between the pathological state of the arterial wall and the information available from the contrast "lumenogram".

Atherosclerosis regression in patients, inferred from improvement in the arteriogram, may occur in several ways. Plaque lipid may be depleted as may its connective tissue. ${ }^{35}$ Lysis of fully occlusive thrombi or of mural thrombi is commonly seen in the course of unstable clinical syndromes. Remodelling of the underlying vascular architecture or relaxation of excess vasomotor tone can improve arteriographic lumen size independently of changes in plaque size. ${ }^{9}$ The role of endothelium and the relations of lipid concentrations to its proper function are proving to be important in many of these processes. ${ }^{10}$

As late as 1987 , only anecdotal evidence suggested that the animal observations could be extended to patients with atherosclerotic disease. Although early arteriographic trials ${ }^{9-11}$ had shown that lipid-lowering treatment reduced the likelihood of progression; regression of disease was rarely observed, and was not found to be increased by diet or by the single-drug treatments tested. A recently published series of randomised clinical arteriographic trials (table) has documented the magnitude of regression, its frequency, and the conditions under which it can occur in patients. In order of their publication they are:

National Heart, Lung, and Blood Institute (NHLBI) type $I^{1213}$ - This pioneering controlled clinical trial was initiated in 1972. One hundred and forty three men and women with 95th percentile LDL cholesterol on screening and at least 90 th percentile LDL on subsequent evaluation, who had some evidence of coronary artery disease, but rela- tively mild symptoms were enrolled. Average baseline LDL cholesterol, HDL cholesterol, and triglycerides were $6 \cdot 2,1 \cdot 0$, and $1.6 \mathrm{mmol} / \mathrm{l}$, respectively (or 237,39 , and $145 \mathrm{mg} / \mathrm{dl}$ ). All patients were given professional dietary counselling and the treatment was randomised to cholestyramine $(24 \mathrm{~g}$ daily) or placebo. In the treated group, LDL cholesterol fell $31 \%$ and HDL cholesterol rose $8 \%$. The interval between angiograms was five years. The angiographic endpoints and clinical benefits of this study, and the studies described below are summarised in the table.

The first Cholesterol-Lowering Atherosclerosis Study (CLAS) enrolled 188 patients who had undergone coronary artery bypass graft surgery; serum cholesterol was 4.7$9.1 \mathrm{mmol} / \mathrm{l}$ at entry, providing an opportunity to investigate the effects of lipid lowering on patients with apparently normal cholesterol. ${ }^{14}$ Average baseline LDL cholesterol, HDL cholesterol, and triglycerides were $4 \cdot 4,1 \cdot 1$, and $1.7 \mathrm{mmol} / \mathrm{l}$, respectively. The treated group was given professional dietary counselling, colestipol ( $30 \mathrm{~g}$ daily), and niacin ( $4 \mathrm{~g}$ daily). The control group received placebos and less intensive dietary counselling. LDL cholesterol fell $43 \%$ in the treated patients and HDL rose $37 \%$. The angiographic interval was two years (table).

Program on Surgical Control of the Hyperlipidemias (POSCH) ${ }^{15}$-In this important technical tour de force 838 patients with a history of acute myocardial infarction were randomly assigned to partial ileal bypass surgery or to "usual care", with pretreatment counselling for all in an American Heart Association Phase II diet. Average baseline LDL cholesterol, HDL cholesterol, and triglycerides were $4 \cdot 6,1 \cdot 0$, and $2 \cdot 3 \mathrm{mmol} / \mathrm{l}$, respectively. In the treated patients LDL cholesterol fell $42 \%$ and HDL rose $5 \%$. Angiograms were done at several points over the 9.7 year follow up; the angiographic findings were reported at baseline and five years (table).

Summary of arteriographic outcomes and frequencies of reported clinical events in eight lipid-lowering trials

\begin{tabular}{|c|c|c|c|c|c|c|c|}
\hline \multirow[b]{2}{*}{ Study } & \multicolumn{3}{|c|}{ Control patients } & \multicolumn{3}{|c|}{ Treatment patients } & \multirow[b]{2}{*}{$\begin{array}{l}\% \text { Event } \\
\text { reduction }\end{array}$} \\
\hline & $\begin{array}{l}\text { Progression } \\
(\%)\end{array}$ & $\begin{array}{l}\text { Regression } \\
(\%)\end{array}$ & $\Delta(\% S) \dagger$ & $\begin{array}{l}\text { Progression } \\
(\%)\end{array}$ & $\begin{array}{l}\text { Regression } \\
(\%)\end{array}$ & $\triangle(\% S)+$ & \\
\hline NHLBI & 49 & 7 & - & 32 & 7 & - & 33 \\
\hline CLAS & 61 & 2 & - & 39 & 16 & - & 25 \\
\hline POSCH (5 yr) & 65 & 6 & - & 37 & 14 & - & 26 \\
\hline Lifestyle+ & - & - & $3 \cdot 4$ & - & - & $-2 \cdot 2$ & $0 v 1$ \\
\hline $\operatorname{FATS}(\mathrm{N}+\mathrm{C})$ & & & & 25 & 39 & $-0.9(0.005)$ & 80 \\
\hline FATS $(\mathrm{L}+\mathrm{C})$ & 46 & 11 & $2 \cdot 1$ & 22 & 32 & $-0.7(0.02)$ & 70 \\
\hline CLAS II & 83 & 6 & - & 30 & 18 & - & 43 \\
\hline UC-SCOR+ & 41 & 13 & $0 \cdot 8$ & 20 & 33 & $-1.53(0.04)$ & $1 v 0$ \\
\hline STARS (D) & & & & 15 & 38 & $-1 \cdot 1(\mathrm{NS})$ & 69 \\
\hline STARS $(D+R)$ & 46 & 4 & $5 \cdot 8$ & 12 & 33 & $-1.9(0.01)$ & 89 \\
\hline Mean & 56 & 7 & $+3 \cdot 0$ & 26 & 26 & -1.4 & \\
\hline
\end{tabular}

* See text for the details, abbreviations and full name of these studies. †Events are variably defined in these studies: in general, the frequency of cardiovascular events (death, myocardial infarction, revascularisation) in control and treated groups are compared using the details and definitions provided. $t \Delta(\% S)$ is usually reported as the average change in percentage stenosis over all the lesions measured per patient. A positive $(+)$ value represents "progression", and a negative $(-)$ value "regression". 
The Lifestyle Heart Trial enrolled 48 patients with documented clinical coronary disease. ${ }^{16}$ Average baseline LDL cholesterol, HDL cholesterol, and triglycerides were $4 \cdot 1,1 \cdot 3$, and $2.4 \mathrm{mmol} / \mathrm{l}$ respectively. The subset randomised to treatment received intense counselling and support in adopting a vegetarian diet with less than $10 \%$ of calories as fat. They were also counselled about relaxation techniques and were encouraged to pursue a moderate exercise regimen. In the treated group LDL cholesterol fell $37 \%$ and HDL fell $3 \%$. The angiographic interval was one year.

Familial Atherosclerosis Treatment Study $(\text { FATS })^{17}$ - One hundred and forty six patients with angiographic coronary disease, raised apolipoprotein $\mathrm{B}$, and a positive family history of coronary heart disease were enrolled. Baseline LDL cholesterol, HDL cholesterol, and triglycerides were $4.9,1 \cdot 0$, and $2.4 \mathrm{mmol} / \mathrm{l}$, respectively. Treatment was randomly assigned; the control group received professional dietary counselling and also colestipol ( $30 \mathrm{~g}$ daily) if the LDL cholesterol was greater than the 90 th percentile for age. Two intensively treated groups received dietary counselling, colestipol, and either lovastatin $40 \mathrm{mg}$ daily or niacin $4 \mathrm{~g}$ daily, with a specified dose increase for failure to achieve the target of LDL cholesterol $<3.1 \mathrm{mmol} / \mathrm{l}$. In the niacin/colestipol group, LDL cholesterol fell $32 \%$ and HDL rose $43 \%$. In the lovastatin/colestipol group, LDL fell $46 \%$ and HDL rose $15 \%$. The angiographic interval was $2 \cdot 5$ years (table).

The second Cholesterol-Lowering Atherosclerosis Study $(C L A S I I)^{18}$ is an extension of the original CLAS study. Baseline LDL cholesterol, HDL cholesterol, and triglycerides averaged $4 \cdot 4,1 \cdot 1$, and $1.8 \mathrm{mmol} / 1$, respectively. One hundred and thirty eight patients re-entered the protocol on the same regimen and 103 eventually completed it at 4 years. LDL cholesterol fell $40 \%$ and HDL cholesterol rose $37 \%$. An angiographic analysis identical to CLAS was used to compare films at baseline and four years. The table shows the results.

University of California Specialized Center of Research project (UC SCOR) ${ }^{19}$ - Ninety seven men and women selected for the genetic disorder familial hypercholesterolaemia were randomly assigned to either intensive lipidlowering therapy or to "usual care" by the current standard of community physicians. Baseline LDL cholesterol, HDL cholesterol, and triglycerides were $7 \cdot 3,1 \cdot 4$, and $1.4 \mathrm{mmol} / 1$ respectively. The intensive regimen included professional dietary counselling, colestipol $30 \mathrm{~g}$ daily, niacin up to $7.5 \mathrm{~g}$ daily, and possibly lovastatin $40 \mathrm{mg}$ daily. LDL cholesterol fell $39 \%$ and HDL cholesterol rose $25 \%$. The angiographic interval was two years. For results see the table.

St Thomas Atherosclerosis Regression Study (STARS)-Ninety patients with clinically diagnosed coronary artery disease and hypercholesterolaemia were recruited. ${ }^{20}$ Baseline LDL cholesterol, HDL cholesterol, and triglycerides averaged $5 \cdot 0,1 \cdot 2$, and $2 \cdot 3 \mathrm{mmol} / 1$ respectively. There were three treatment groups: the control regimen was randomisation to "usual care". Two more intense approaches include professional dietary counselling and dietary counselling plus cholestyramine (16 g daily). Among patients $\frac{T}{\mathbb{T}}$ receiving dietary counselling, LDL cho- $\frac{\varrho}{\beth}$ lesterol fell $16 \%$ and HDL cholesterol was $\stackrel{.}{.}$ unchanged. Among those receiving cho- $\Rightarrow$ lestyramine and diet, LDL cholesterol fell $36 \%$ and HDL cholesterol fell $4 \%$. The 등 angiographic interval was three years. See the $\frac{\overline{\bar{m}}}{\bar{m}}$ table for results.

In the NHLBI II, CLAS, CLAS II, and $\stackrel{\curvearrowright}{\varrho}$ POSCH trials change in arterial obstruction कs was assessed visually by panels of experts $\vec{O}$ blinded to patient identity, randomisation, $\overrightarrow{\vec{\omega}}$ and temporal sequence of the film pair. In the ${ }_{\sigma}$ remainder of the trials a similarly blinded analysis incorporated techniques of computer-assisted quantitative arteriography. ${ }^{721}$ In only four of these eight trials of lipid-lowering $(s)$ therapy was there an entry requirement for even modest hyperlipidaemia. Despite the diversity among these studies in clinical pre- $D$ sentation, lipid entry requirements, treatment ${ }_{0}^{\infty}$ regimens and methods for arteriographic analysis, their outcomes, summarised in the $\vec{c}$ table, are surprisingly consistent. Each study demonstrated an arterial benefit from treatment, be it diet or diet supplemented by ileal ${ }^{\curvearrowright}$ bypass surgery or by other lifestyle changes or $\vec{\oplus}$ by lipid-altering drugs. As a generalisation $\omega$ from these results, less than a twelfth of the control group patients were found to have favourable changes in arterial obstruction ("regression") during the study period. By contrast, more than a quarter of treated $\varrho$ patients were found to have overall regression $\overrightarrow{\vec{O}}$ (a 3-4-fold increase). The table shows that 3 mean estimates of coronary disease severity per patient worsened (progressed) by about $3 \%$ stenosis among the controls whereas there was an improvement (regression) by $1-2 \%$ stenosis among the treated patients. In nearly every study the frequency of cardiovascular events was reduced substantially by $\frac{0}{\partial}$ treatment although the event reductions achieved statistical significance in only three. $\frac{\rho}{5}$ Failure to confirm statistically a clinical bene- $\frac{D}{2}$ fit is not unexpected because the sample size required for trials attempting to demonstrate $N^{\circ}$ arteriographic benefits is considerably smaller $N$ than for trials to demonstrate clinical benefits. N్

\section{REGRESSION AMONG LESIONS}

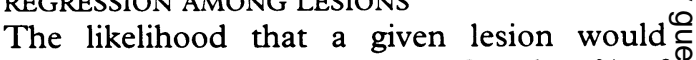
regress was very low. In FATS only $5 \%$ of $\stackrel{\Phi}{+}$ lesions in the control group and $12 \%$ in the treated group improved by the criterion

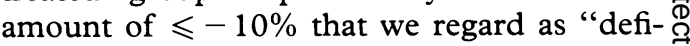
nite" regression by our method. ${ }^{21}$ Lesions $\stackrel{Q}{\varrho}$ were classified as mild, moderate, or severe, depending on the severity of the stenosis at 8 baseline. Figure 1 shows the importance of lesion severity and of treatment on progression of individual lesions. In general, progression and regression are both more frequent in more severe lesions. Intensive lipid-lowering therapy doubles the likelihood of regression 


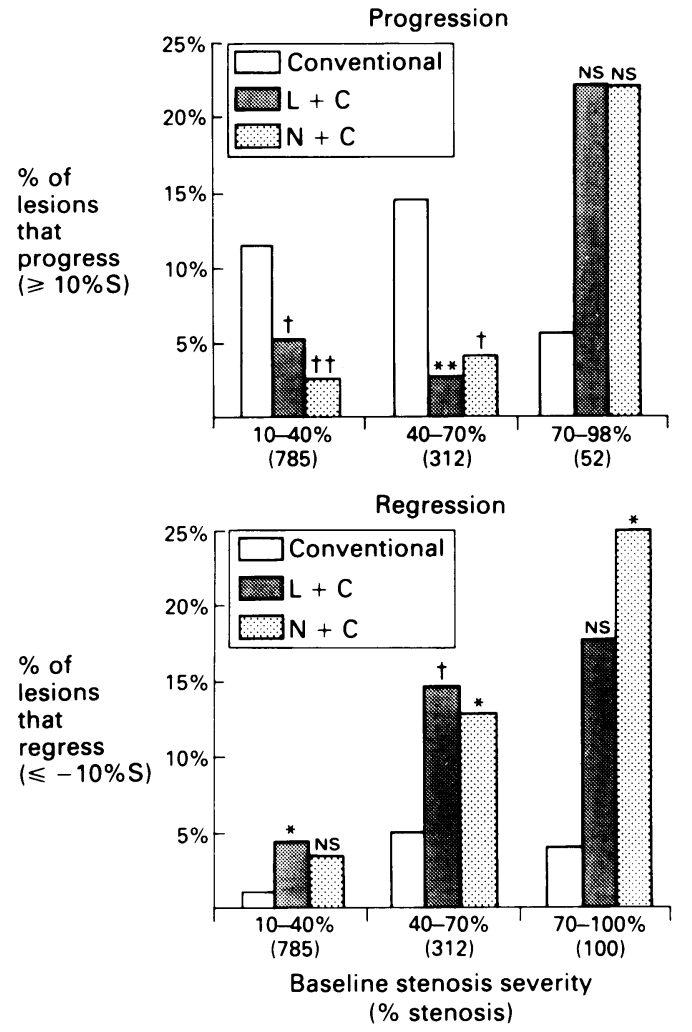

Figure 1 Frequency of definite lesion regression in FATS expressed as the percentage of lesions that decrease in severity (regress) by a measured $10 \%$ stenosis or more. Lesions from 120 patients are subgrouped into 785 mild stenoses $(10 \%-40 \% S), 312$ moderate stenoses

$(40 \%-70 \% S)$, and 52 severe stenoses $(70 \%-98 \%$ S). Also all 48 initially totally occluded lesions are added to the severe lesion regression analysis, because these may "regress" by recanalisation. In general, change is relatively infrequent. The more severe the lesion at baseline, the more likely it is to change. Intensive lipid-lowering therapy increases regression frequency at all levels of severity and reduces progression frequency for mild and moderate lesions. $\chi^{2}$ statistical comparisons versus control group

(conventional treatment) frequency: ${ }^{\star} p<0.05$;

$t p<0.02 ;^{* *} p<0.001 ;++p<0.005$. L, lovastatin;

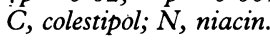

among mild and moderate lesions, and quadruples it among the severe lesions. The small number of severe lesions in this study (52 in the $70-98 \%$ range and 48 total occlusions) precludes confident statements about the effect of therapy on change in severe lesions.

Thus very few lesions undergo natural or spontaneous regression. Though this number can be increased significantly by lipid-lowering therapy most lesions seem to be unaltered by treatments that can be characterised as "intensive" and result in considerable favourable alterations in the lipid and lipoprotein profile. Paradoxically, these regimens are commonly associated with substantial reductions in clinical event rate (table). We will return to this apparent paradox below.

RETARDING PROGRESSION OF CAD

Support for the idea that lipid-lowering therapy can effectively retard progression of atherosclerotic arterial obstruction dates from 1979. ${ }^{11}$ The composite of such evidence from randomised arteriographic trials is summarised in the table. Again, despite the diversity of these trials, the evidence for reduced disease progression with therapy is suprisingly consistent. As a generalisation from the table over a half of the control group but only a quarter of treated patients were judged to have worsening arterial obstruction during the study periods. On a per-lesion basis, lesion progression among mild and moderate lesions is about $75 \%$ less likely with intensive therapy. Progression of severe lesions seems unaltered by intensive therapy: the numbers on which this result is based are small, however.

\section{Preventing plaque disruption and clinical events}

PREVENTION OF CLINICAL EVENTS

The landmark Lipid Research Clinics Program coronary primary prevention trial ${ }^{22}$ established that modest LDL cholesterol reduction promoted a significant reduction in clinical coronary events, but not in total mortality. Importantly, the magnitude of cardiovascular benefit correlated with the amount of LDL cholesterol reduction. The Helsinki Heart Study, ${ }^{23}$ also with modest LDL change, achieved a significant reduction in total cardiac events, but not mortality. The Coronary Drug Project, when updated about eight years after its completion, showed an $11 \%$ reduction in total mortality among those treated with niacin. ${ }^{24}$

Additional evidence that clinical events are decreased by lipid-lowering therapy is seen in the table. Many of these recent trials have used more potent lipid-lowering agents or combination treatments. As a result the magnitude of benefit is correspondingly greater. Each trial reports events somewhat differently. In general, when reported, we have included cardiac death, confirmed myocardial infarction, and progressive, medically refractory ischaemia requiring revascularisation (bypass or angioplasty) among the composite of clinical events. Figure 2 shows data from the FATS trial. It is thus clear from the table and fig 2 and from the primary prevention trials that clinical cardiovascular events are reduced by lipid-lowering therapy. The magnitude of reduction in clinical risk seems out of keeping with the average $1-2 \% \mathrm{~S}$ diameter stenosis regression in lesion severity and with the fact that only about $12 \%$ of all intensively treated lesions actually regress by a measurable amount. To understand how regression of a small number of lesions might result in a roughly $60 \%$ (range $25 \%-89 \%$ ) reduction in the frequency of clinical events, we must understand the series of events in the plaque that turn a stable quiescent lesion into an unstable ischaemia-provoking culprit lesion.

DETERMINANTS OF PLAQUE DISRUPTION

Two evolving insights have altered our understanding of the precipitation of clinical coronary events. First, mild and moderate coronary lesions $(<70 \%$ stenosis $)$ may abruptly progress to severe obstruction, with resulting unstable angina, myocardial infarction, or death. In fact, most clinical events 


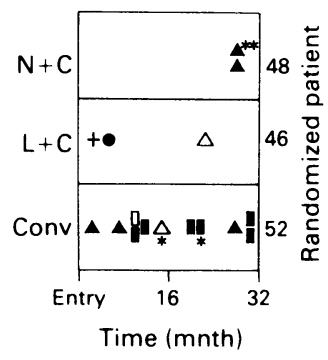

Event symbols:

- coronary angioplasty; $\triangle$ peripheral angioplasty;

I coronary bypass:

n peripheral bypass

- myocardial infarction:

+ cardiac death

Figure 2 Timing of clinical cardiovascular "events" (death, myocardial infarction, or revascularisation for patients in each of the treatment strategies of FATS. This "intention to treat" analysis includes all patients randomised to each group. $L+C$ and $N+$ $C v$ conventional treatment (Conv): $p<$ $0.01 . N$, niacin; $C$ colestipol; L, lovastatin. ${ }^{\star}$ One patient had two events. **This patien left the study after four months. worsening ischaemia) in three randomised

occur under these circumstances. ${ }^{25} 26$ Specifically, when the lesion precipitating a myocardial infarct has, by chance, been visualised on a recent angiogram, its preinfarct severity averages $50 \%$ stenosis, and it will not usually possess features indicating that it is destined soon to become occluded. ${ }^{25}{ }^{26}$ A second, histological, insight is that most ischaemic coronary events are linked to a "culprit" lesion with one or more of the following features at histological examination: (a) a fissured, torn, or vented fibrous cap; (b) mural thrombus adherent at the site of the fissure; $(c)$ bleeding into the core lipid region; and $(d)$ severe arterial obstruction secondary to the composite mass of expanded plaque and thrombus.

Figure 3 shows angiographic images of a plaque that has become unstable and caused a myocardial infarction. How does an "ordinary" coronary lesion become so disruptively transformed? Serial histological sectioning of such an acutely occluded coronary lesion will inevitably show a site of fissuring of the fibrous cap that overlies the lipid-rich core of the atheroma. ${ }^{27} 28$ This fissure is the nidus from which occlusive thrombus has grown, and it can be the point of entry of haemorrhage into the plaque. Certain plaque characteristics increase the risk of disruption. In fibrous caps that fissure, the foam cell density is greater than that in intact caps. ${ }^{27-29}$ Furthermore, the most common site of fissuring occurred at the shoulder of an eccentric lipid-rich plaque, a location of high foam cell density $^{29}$ and also high circumferential shear stress, according to a computer model of repeated cyclic distension of the diseased arterial cross section. ${ }^{30}$ Fissuring of the fibrous cap was observed and predicted to occur more frequently at the shoulders of lipid pools occupying more than $15 \%$ of the arterial circumference. ${ }^{30}$ In $83 \%$ of 86 infarct lesions the intimal fissure extended from the lumen into an unstructured pool of extracellular lipid. ${ }^{30}$

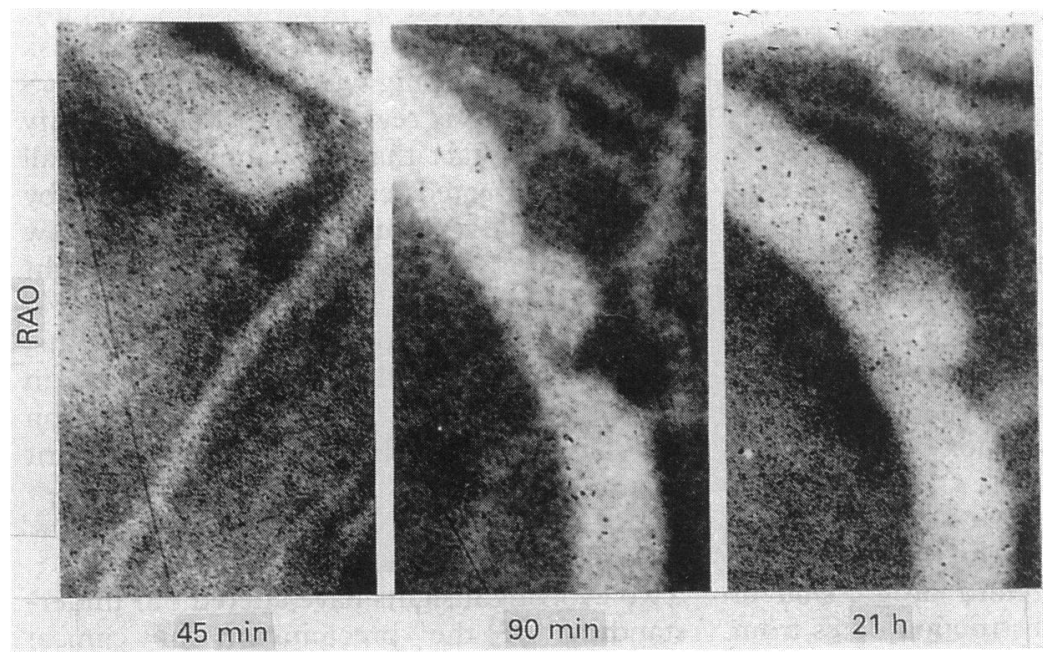

Figure 3 Angiographic appearance of a lesion of the left circumflex coronary artery in a patient presenting with acute myocardial infarction and treated with alteplase (tPA). When the artery opened 90 minutes later, a contrast-filled pocket was revealed. This morphology suggests haemorrhage through a fissure, or through plaque microvessels, into the lipid-rich core of the plaque, forcing its thin fibrous cap into the lumen and abruptly worsening obstruction.
Thus the histological features of a large lipid pool and of an abundance of lipid laden foam cells in the fibrous cap and shoulders of the atheroma each predispose to plaque $\underline{\underline{T}}$ fissuring, subsequent plaque haemorrhage $I$ and disruption, and to coronary events.

\section{PREVENTION OF PLAQUE DISRUPTION}

Reduction of plasma LDL cholesterol might be expected to reduce the likelihood of fissur- $\bar{c}$ ing because of the known favourable effects of $\underline{\underline{n}}$ LDL cholesterol reduction on the above pre- $\frac{\bar{D}}{\vec{D}}$ dictors. $^{2-5}$ A consequence of such protection $\triangle$ against fissuring would be a decline in the frequency of abrupt progression to clinical $\overrightarrow{0}$ events among patients in whom LDL cholesterol has been therapeutically reduced. $\vec{\omega}$ Indeed, this has been the case. Figure $2 \stackrel{2}{\partial}$ shows that in FATS there were 13 coronary and three peripheral vascular events (cardiac death, myocardial infarction, or revascularisation (PTCA or CABG) for refractory progressive ischaemia) among 15 patients. The decision for revascularisation was indepen- is dent of the FATS trial investigators: it was $\infty$ made by private physicians who were blinded 윽 to randomisation and to the study lipid $\vec{c}$ response. Analysis of the 13 coronary events among the 146 patients ${ }^{17}$ showed that all clinical events were associated with a culprit $\underset{2}{2}$ coronary lesion located in the vascular distri- $\vec{\bullet}$ bution of the ischaemic electrocardiographic $\omega$ changes. Each had progressed significantly in severity from the baseline stenosis measurement to that at the time of the event. Furthermore, the progression of mild and moderately narrowed lesions to clinical events $\stackrel{2}{\mathbb{2}}$ was virtually abolished by lipid-lowering $\underset{\Rightarrow}{\vec{A}}$ therapy (fig 4). Eight of nine clinical events 을 among the conventionally treated patients arose from lesions initially less than $70 \%$ 훙 diameter stenosis (S) (baseline mean $=$

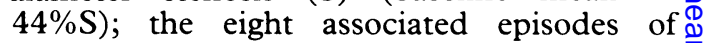
plaque disruption occurred in a pool of $414 \stackrel{7}{7}$ mild or moderate lesions. Among patients in the two intensively treated groups, only one of 683 mild or moderate lesions progressed to an event $(p<0.005$, per patient; $p<0.02$ per 0 lesion). By comparison, severe lesions $(\geq 70 \% \mathrm{~S})$ did not appear to so benefit from lipid-lowering: the numbers on which this statement is based are small, however.

\section{Summary}

Lipid-lowering therapy, as assessed by@ angiography, clearly benefits the arterial dis- $\stackrel{\mathbb{D}}{\mathscr{D}}$ ease process. For example, among intensively treated patients in FATS the frequency of definite progression per lesion at risk was $\overrightarrow{\mathbb{D}}$ reduced by $75 \%$ among mild and moderate $\frac{\rho}{\Phi}$ lesions, which form the great preponderance $\frac{\Omega}{\sigma}$ of the lesion population. Regression frequency per lesion was more than doubled by융 intensive therapy in mild and moderate subgroups and quadrupled in the subgroup with $\frac{0}{7}$ severe lesions. Clinical events were reduced by $73 \%$. This was clearly due to a 15 -fold reduction in the likelihood that a mildly or moderately diseased arterial segment would 


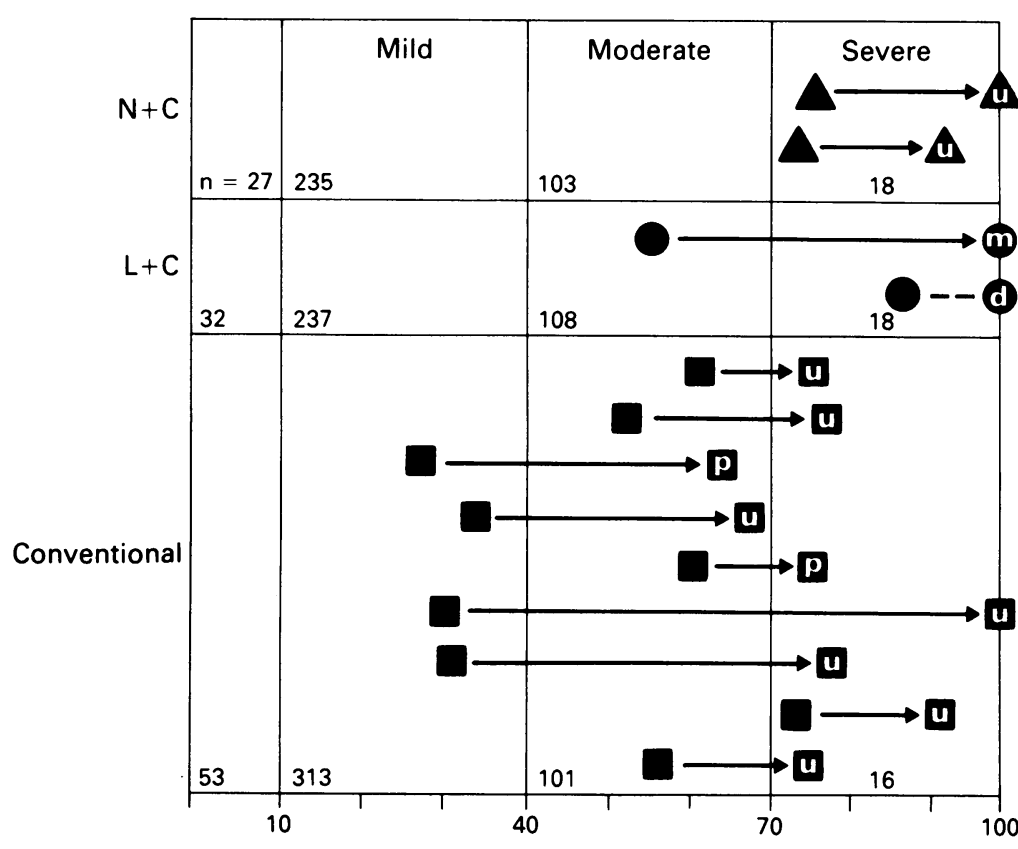

Stenosis severity

(Baseline $\rightarrow$ event) $(\% S)$

Figure 4 Lesion changes associated with the thirteen coronary events as measured from 1316 lesions in 120 FATS patients. Among lesions exposed to intensive lipid-lowering therapy, only one of 638 lesions that were mild to moderate at baseline among 74 such patients progressed to a clinical event whereas eight of 414 such lesions among 46 conventionally treated patients did so (per patient, $p<0.005$; per lesion $p<0.02$ ). By this standard, severe lesions did not seem to benefit from therapy. $N$, niacin; $C$, colestipol; $L$, lovastatin; Conv, conventional therapy. Events: $U$, unstable angina; $M$, myocardial infarction; $D$, death; $P$, progressive angina. $\% S$, percentage diameter stenosis. The number 55 in each panel represents the number of lesions at risk of progressing at baseline in each subgroup.

undergo abrupt and substantial progression to a severe lesion at the time of the clinical event. It has been shown that the process of plaque fissuring, leading to plaque disruption, thrombosis, and clinical coronary events, is predicted by the size of the plaque core lipid pool and the abundance of lipid-laden macrophages in its fibrous cap. Experimentally, lipid lowering therapy decreases the number of lipid-laden intimal macrophages and more slowly depletes core cholesteryl ester deposits. Thus the composite of new and previously published data presented here supports the idea that lipid-lowering therapy selectively lipid-depletes (causes regression of) those fatty lesions containing a large lipid core and abundant intimal foam cells. By doing so, these lesions, which are most vulnerable to fissuring, are rendered much more stable and the clinical event rate is accordingly decreased.

We thank Ellen Zincavage and Betsy Sayler for their help in preparing and researching this paper.

Supported in part by NIH Grants R01 HL19451, P01 HL 30086, and R01 HL 42419 from the National Heart, Lung, and Blood Institute and in part by a grant from the John L Locke, JR Charitable Trust, Seattle, WA.

1 Wissler RW, Vesselinovitch D. Can atherosclerotic plaques regress? Anatomic and biochemical evidence from nonhuman animal models. Am $\mathcal{f}$ Cardiol 1990 65:33-40.

2 Armstrong ML, Megan MB. Lipid depletion in atheromatous coronary arteries in rhesus monkeys after regression diets. Circ Res 1972;30:675-80.

3 Clarkson TB, Bond MG, Bullock BC, Marzetta CA. A study of atherosclerosis regression in Macaca mulatta. IV. Changes in coronary arteries from animals with atherosclerosis induced for 19 months and then regressed for 24 or 48 months at plasma cholesterol concentrations of 300 or $200 \mathrm{mg} / \mathrm{dl}$. Exp Mol Pathol 1981;34: 345-68.

4 Small DM, Bond MG, Waugh D, Prack M, Sawyer JK Physiochemical and histological changes in the arterial wall of nonhuman primates during progression and regression of atherosclerosis. f Clin Invest 1984;73: 1590-1605.

5 Armstrong MC, Megan MB. Arterial fibrous protein in cynomolgus monkeys after atherogenic and regression diets. Circ Res 1975;36:256-61.

6 Gerrity RG. The role of monocyte in atherogenesis. I. Transition of blood-borne monocytes into foam cells in fatty lesions.

7 Kirkeeide RL, Gould KL, Parsel I. Assessment of coronary stenoses by myocardial perfusion imaging during pharmacologic coronary vasodilation. VI. Validation of coronary flow reserve as a single integrated functional measure of stenosis severity, reflecting all its geometric dimensions. F Am Coll Cardiol 1986;7:103-13.

8 Brown BG. What the angiogram does reveal about atherosclerosis morphology, progression, and regression. In: Tobis JM, Yock PG, eds. Intravascular ultrasound imaging. New York: Churchill Livingstone, 1992: imaging.

9 Glagov S, Weisenberg E, Zarins CK, Stankunavicius R, Kolettis GJ. Compensatory enlargement of human atherosclerotic coronary arteries. $N$ Engl f Med 1987;316: $1371-5$

10 Vita JA, Treasure CB, Nabel EG, McLenachan JM, Fish RD, Yeung AC, Vekstein VI, Selwyn AP, Ganz P. Coronary vasomotor response to acetylcholine relates to risk factors for coronary artery disease. Circulation 1990;81:491-7.

11 Kuo PT, Hayase K, Kostic JB, Moreyra AB. Use of combined diet and colestipol in long-term (7-7.5 years) treatment of patients with Type II hyperlipoproteinemia. Circulation 1979;59:199-214.

12 Brensike JF, Levy RI, Kelsey SF, et al. Effects of therapy with cholestyramine on progression of coronary atherosclerosis: Results of the NHLBI Type II coronary intervention study. Circulation 1984;69:313-24.

13 Levy RI, Brensike JF, Epstein SE, Kelsey SF, Passamoni ER, Richardson JM, et al. The influence of changes in lipid values induced by cholestyramine and diet on progression of coronary artery disease: Results of the NHLBI type II Coronary Intervention Study. Circulation 1984;69:325-37.

14 Blakenhorn DH, Nessim SA, Johnson RL, Sanmarco ME, Azen SP, Cachin-Hamphill L. Beneficial effects of colestipol niacin therapy on coronary atherosclerosis and coronary venous bypass grafts. $\mathfrak{F} A M A 1987 ; 257$ : and coro.

15 Buchwald H, Varco RL, Matts JP, Long JM, Fitch LL, Campbell GS, et al. Effect of partial ileal bypass on mortality and morbidity from coronary heart disease in patients with hypercholesterolemia-Report of the Program on Surgical Control of the Hyperlipidemias (POSCH). N Engl f Med 1990;323:946-55.

16 Ornish $\mathrm{D}$, et al. Can lifestyle changes reverse coronary heart disease? Lancet 1990;336:129-33.

17 Brown BG, Albers JJ, Fisher LD, et al. Regression of coronary artery disease as a result of intensive lipid-lowering therapy in men with high levels of apolipoprotein B. N Engl ₹ Med 1990;323:1289-98.

18 Cashin-Hemphill L, Mack WJ, Pogoda MJ, Sanmarco ME, Azen SP, Blankenhorn DH. Beneficial effects of colestipol-niacin on coronary atherosclerosis. $¥ A M A$ 1990;264:3013-7.

19 Kane JP, Malloy MJ, Ports TA, et al. Regression of coronary atherosclerosis during treatment of familial hypercholesterolemia with combined drug regimens. $\mathfrak{f} A M A$ 1990;264:3007-12.

20 Watts GF, Lewis B, Brunt JNH, Lewis ES, et al. Effects on coronary artery disease of lipid-lowering diet, or diet plus cholestyramine, in the St. Thomas' Atherosclerosis Regression Study (STARS). Lancet 1992;339:563-9.

21 Brown BG, Bolson EL, Dodge HT. Quantitative computer techniques for analyzing coronary arteriograms. Prog Cardiovasc Dis 1986;28:403-418.

22 The Lipid Research Clinics Program: The Lipid Research Clinics Coronary Primary Prevention Trial results: I. Clinics Coronary Primary Prevention Trial results: I. Reduction in incidence
$\Im A M A 1984 ; 251: 351-64$.

23 Manninen V, Elo MO, Frick MH, et al. Lipid alterations and decline in the incidence of coronary heart disease in the Helsinki Heart Study. $¥ A M A$ 1988;260:641-51.

24 Conner PL, Berge KG, Wenger NK, et al. Fifteen year mortality in Coronary Drug Project patients: long-term benefit with niacin. 7 Am Coll Cardiol 1986;8:1245-55.

25 Brown BG, Gallery CA, Badger RS, Kennedy JW Mathey D, Bolson EL, Dodge HT. Incomplete lysis of thrombus in the moderate underlying atherosclerotic lesion during intracoronary infusion of streptokinase for acute myocardial infarction: Quantitative angiographic acute myocardial infarction: Quantitative

26 Little WC, Constantinescu M, Applegate RM, et al. Can coronary angiography predict the site of a subsequent myocardial infarction in patients with mild-to-moderate coronary artery disease? Circulation 1988,78.1157-66.

27 Constantinides $P$. Plaque fissures in human coronary thrombosis. I Atheroscler Res 1966;61:1-17.

28 Constantinides P. Plaque hemorrhages, their genesis and their role in supra-plaque thrombosis and atherogenesis. In: Glagov S, Newman WP, Schaffer SA, eds. Pathobiology of the human atherosclerotic plaque. New York: Springer Verlag, 1989:393-411.

29 Lendon CL, Davies MJ, Born GVR, Richardson PD. Atherosclerotic plaque caps are locally weakened when macrophage density is increased. Atherosclerosis 1991 87:87-90.

30 Richardson PD, Davies MJ, Born GVR: Influence of plaque configuration and stress distribution on fissuring of coronary atherosclerotic plaques. Lancet 1989;ii: 941-44. 